\title{
Dissociating what and when of intentional actions
}

\author{
Veronika Krieghoff ${ }^{1}$, Marcel Brass ${ }^{1,2}$, Wolfgang Prinz ${ }^{1}$ and Florian Waszak ${ }^{3}$ \\ 1 Department of Psychology, Max-Planck-Institute for Human Cognitive and Brain Sciences, Leipzig, Germany \\ 2 Department of Experimental Psychology and Ghent Institute for Functional and Metabolic Imaging, Ghent University, Ghent, Belgium \\ ${ }^{3}$ Laboratoire Psychologie de la Perception, UMR CNRS 8158, Université Paris Descartes, Paris, France
}

Edited by:

Silvia A. Bunge, University of California Berkeley, USA

Reviewed by:

Deborah Serrien, University of

Nottingham, UK

Silvia A. Bunge, University of California

Berkeley, USA

${ }^{*}$ Correspondence:

Veronika Krieghoff, Max-Planck-

Institute for Human Cognitive and Brain

Sciences, Stephanstr. 1a, 04103

Leipzig, Germany.

e-mail:veronika.krieghoff@cbs.mpg.de
Recent brain imaging research revealed that internally guided actions involve the frontomedian wall, in particular the preSMA and the rostral cingulate zone (RCZ). However, a systematic decomposition of different components of intentional action is still lacking. We propose a new paradigm to dissociate two components of internally guided behavior: Which action to perform (selection component) and when to perform the action (timing component). Our results suggest a neuro-functional dissociation of intentional action timing and intentional action selection. While the RCZ is more strongly activated for the selection component, a part of the superior medial frontal gyrus is more strongly activated for the timing component. However, in a post hoc conducted signal strength analysis we did also observe an interaction between action timing and action selection, indicating that decisional processes concerning action timing and action selection are not completely dissociated but interdependent. Altogether this study challenges the idea of a unitary system supporting voluntary action and instead suggests the existence of different neuroanatomically dissociable subfunctions.

Keywords: fMRI, intentional action, action timing, action selection, frontal medial wall

\section{INTRODUCTION}

Human actions exist along a continuum with regard to whether they are externally or internally controlled. In every day life, actions rarely correspond to the extremes of the continuum. Nevertheless, they can be classified into actions guided foremost by internal factors, for example switching on TV to watch the news, and actions guided rather by environmental stimuli, for example stopping in front of a red traffic light.

The two types of actions differ in functional terms and in the neurophysiological substrate that controls the actions. Herwig et al. (2007) suggest that, in the stimulus-based mode, the agent passes on control to the environment in that the system merely acts upon presentation of a particular stimulus in a prespecified way (prepared reflex, see Hommel, 2000). These actions are selected with respect to their sensory antecedents. By contrast, in the intentionbased mode actions are guided by the ideomotor principle, that is, they are selected with respect to the intended sensory consequences (e.g., Greenwald, 1970; Prinz, 1997). Regarding the underlying neuroanatomical differences between the two action modes (Goldberg, 1985) emphasized the distinction between a medial and a lateral motor system which are involved in internally and externally guided actions, respectively.

As concerns voluntary actions, fMRI studies show that several areas in the frontomesial cortex including the preSMA and the anterior cingulate cortex (ACC) play a major role in the internal selection and timing of action, as well as in related functions like the inhibition of competing action alternatives and the evaluation of likely outcomes and rewards of actions (e.g., Cunnington et al., 2002, 2005; Lau et al., 2004a,b, 2006; Rushworth et al., 2004). However, performing a voluntary action necessitates a large amount of information to be generated and processed. The areas involved in voluntary action control are not only in charge of the prediction of the sensory consequences of the action (see Blakemore et al., 1998) and of the conscious experience of intending to act (see Haggard and Clarke, 2003), they also have to take care of a series of decisions regarding whether to act (see Brass and Haggard, 2007), what action to perform and when to perform it. It is at present unclear how precisely these different cognitive antecedents of voluntary actions are controlled in the brain.

The present study is meant to fill one of the gaps in our current knowledge of the neurophysiological substrate of voluntary action control. To be precise, we aimed at providing further insights into the role of frontomesial cortical regions in two different decision processes that are basic for carrying out a voluntary action: When people perform voluntary actions, they do not only have to choose which action to perform, they also have to determine an appropriate point in time to perform it. Imagine a soccer player approaching the goal. He has to decide whether to pass the ball or to shoot on the goal. He also has to choose the right moment to act. Thus, there are at least two components of internally guided actions: 'what' and 'when' (see Mueller et al., 2007). In the following we label actions referring to the 'when-component' internally or externally timed, whereas we label actions referring to the 'what-component' internally or externally selected.

Usually studies on voluntary action investigate one or the other component of voluntary action control. A direct comparison of both components within one paradigm has never been carried out. Typically, in studies exploring internal action timing, a condition in which subjects self-initiate a key press is compared with a condition in which subjects respond to a visual or acoustic cue (c.f. Deiber et al., 1999). As a correlate of internal action timing those studies reported activation in the preSMA and/or cingulate motor 
areas (Cunnington et al., 2002; Debaere et al., 2003; Deiber et al., 1999; Jahanshahi et al., 1995; Jenkins et al., 2000; Wiese et al., 2004). Similar activations were found in studies exploring the internal selection of actions (Cunnington et al., 2006; Lau et al., 2006; van Eimeren et al., 2006). van Eimeren et al. (2006), for example compared a full-selection condition in which subjects could freely select which one out of four buttons to press with different restricted conditions (two or three response possibilities) as well as with a full restricted condition (forced choice). The most prominent activation they found as a correlate for internal action selection was located in the ACC, and the preSMA. Taken together there seems to be no evidence for a specific activation related to the selection or timing component of intentional action. This finding is in line with the conclusion (Jahanshahi and Frith, 1998) drew from a review of several neuroimaging studies: They found several cortical (dorsolateral prefrontal cortex, ACC and SMA) and subcortical structures (thalamus and basal ganglia) to be involved in the control of voluntary actions regardless of whether they are internally selected, timed or inhibited and proposed the existence of a uniform 'willed action system'.

However, contrary to this notion, Mueller et al. (2007) provided evidence for a dissociation of the 'what-' and the 'when-component' of intentional action, using a paradigm introduced by Waszak et al. (2005). In this paradigm, subjects performed a temporal bisection task, making left or right key presses at the midpoint between isochronous pacing signals (a sequence of ' $X$ 's presented to the left or the right of the fixation cross). In the internally selected condition the subjects' key press determined the location of the subsequent ' $\mathrm{X}$ ' on the screen. In this condition, subjects were instructed to generate a random sequence of left and right 'X's. In the externally selected condition, by contrast, the subjects' key press was prompted by the location of the preceding stimulus. The movements in a given externally selected run were yoked (in a disguised fashion) to the movements produced in the preceding internally selected run. The results of the study by Mueller et al. (2007) revealed that the rostral cingulate zone (RCZ) but not the preSMA was related to internal action selection. Mueller and colleagues speculated that the preSMA might be more closely linked to the internal action timing. However, a direct comparison of both components - necessary to demonstrate a dissociation between the two aspects of action control within the frontomedian wall - has never been carried out.

In the present study we directly tested for a functional dissociation of 'when-' and 'what-component' within regions of the frontomedian wall. To do so, we independently manipulated these two components of voluntary action control within one task. Subjects were instructed to decide as fast as possible after presentation of a cue which action to perform, and when. The two decisions were either taken by the participants (internal) or based on the cue (external), enabling us to differentiate between internal and external 'when' and 'what' decisions. Based on the findings of Mueller et al. (2007), we expected to find increased activation in the RCZ for internally selected actions and increased activation in the preSMA for internally timed actions. In this previous study we could show a similar network of activations for internally and externally selected actions, but to a stronger degree for internally selected actions. From this result we concluded that externally guided actions are generally less complex than internally guided actions. Therefore we did not expect to find activation differences for the main effects of the reversed contrasts (externally selected and externally timed actions respectively).

While the primary goal of our study was to investigate brain areas that are involved in the decision processes that occur in the cue phase, we tested whether frontomedian activation was also related to the implementation of the action. In our paradigm the moment when the subjects have to make the decision what to do, and when, and the moment when the subjects actually have to execute the action were experimentally dissociated. Subjects were instructed to decide as fast as possible after cue presentation which action to perform, and when. However, it is only after a variable cuetarget-interval (CTI) that they were required to perform the action, enabling us to separate cue and target BOLD-response (reflecting decisional processes and processes related to the implementation of the action, respectively).

We assumed that activation differences in the frontomedian wall reflect mainly decisional aspects of voluntary action control. Since we were especially interested in these 'what' and 'when' decisions, the fMRI analysis focused on activations upon cue presentation. By contrast, we presumed that the implementation of the decision should not differentially involve frontomedian brain regions. Thus we hypothesized to find no activation differences contingent on the target in the frontomedian wall. The data of the target period are also presented below.

\section{MATERIALS AND METHODS SUBJECTS}

Sixteen healthy subjects (eight females) with a mean age of 25.44 years ( $\mathrm{SD} \pm 2.87$ ) with normal or corrected to normal vision participated in the study. Two subjects were excluded from the analysis because they were not able to synchronize key presses with the pacing signals. Hence, 14 subjects remained (6 females). All subjects were right-handed as indicated by scores on the Edinburgh Handedness Inventory (Oldfield, 1971). Subjects gave written informed consent to the study. All subjects had extensive experience with participating in fMRI studies and had no history of psychiatric, major medical, or neurological disorder.

\section{TASK}

The sequence of events in one trial was as follows (see Figure 1). At the beginning of each trial subjects were presented with a cue consisting of two letters. After a variable CTI (jittered in a pseudologarithmic order in steps of $400 \mathrm{~ms}$ from 200 to $10400 \mathrm{~ms}$ ), during which subjects viewed a fixation cross at the center of the screen, four tones (duration $=100 \mathrm{~ms}$ ) were presented at a constant interval of $1000 \mathrm{~ms}$. The rationale behind the intense jittering of the CTI was to separate cue-related activation from target-related activation. This separation was critical to the present study as we wanted to look at processes underlying action planning rather than action execution. Furthermore it is important to note that we introduced four target tones rather than two target tones to assure that subjects could prepare the two possible action times equally well. This manipulation also guarantees that subjects responded with the tone rather than reacted on the tone as they were able to anticipate the appearance of the target tone. The task of the subject was to perform a left or a right key press on the third or the fourth tone. 


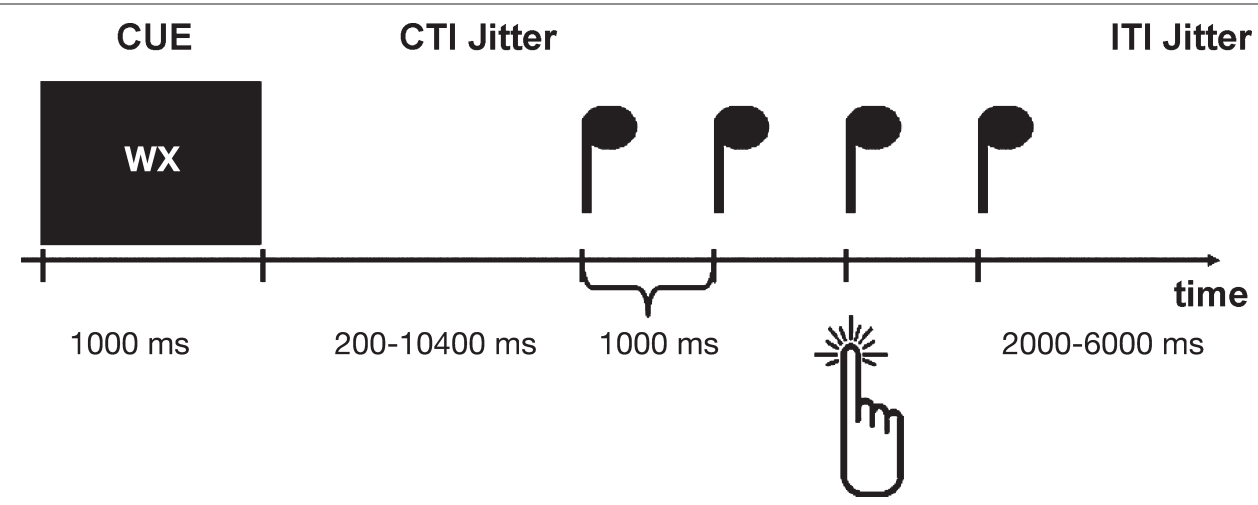

FIGURE 1 | Paradigm. Experimental design. Sequence of events in one trial. For details see text. First, subjects are presented with a cue indicating an internal or an external what (left or right key press) or when decision (third or fourth tone). In the example subjects could freely select which button to press, and when. After a variable CTI (jittered in a pseudo-logarithmic order in steps of
$400 \mathrm{~ms}$ from 200 to $10400 \mathrm{~ms}$ ), during which subjects viewed a fixation cross at the center of the screen, four tones (duration $=100 \mathrm{~ms}$ ) were presented at a constant interval of $1000 \mathrm{~ms}$. The subjects had to press the appropriate button in synchrony with the appropriate tone. After the tones there was a variable intertrial-interval (ITI) that was jittered in steps of 500 ms from 2000 to 6000 ms.
The first letter of the cue informed the subject about the 'whatdecision': it indicated either that the subject was to freely choose between left and right (W), or it indicated which key to press [L or $\mathrm{R}$ (left/right in German)]. That is the first letter indicated either an internal 'what-decision' or an external 'what-decision'. The second letter of the cue informed the subject about the 'when-decision': it indicated either that the subject was to freely choose between the third and the fourth key press (X), or it indicated the tone on which the subject had to press the key [D or V (third/fourth in German)]. That is the second letter indicated either an internal 'when-decision' or an externally cued 'when-decision'. Subjects were instructed to decide as fast as possible after the cue presentation which action to perform, and when.

Both factors ['what' (externally vs. internally) and 'when' (externally vs. internally)] were independently crossed, resulting in four action conditions: both parameters internal (entirely internal condition), 'what' internal and 'when' external (internal selection condition), 'what' external and 'when' internal (internal timing condition), and 'what' and 'when' external (entirely external condition): After the tones there was a variable inter-trial-interval (ITI) that was jittered in steps of $500 \mathrm{~ms}$ from 2000 to $6000 \mathrm{~ms}$. Due to the intense jittering the total trial length varied between $6600 \mathrm{~ms}$ (for the shortest trial) and $20800 \mathrm{~ms}$ (for the longest trial).

We conducted an event-related-design and stimuli were presented in randomized order. For each of the four conditions there were 36 trials; the whole experiment lasted about $30 \mathrm{~min}$. Prior to the scanning session subjects underwent a training session outside the scanner to become familiar with the paradigm. The procedure was identical to the main session with the exception that we introduced feedback after the button press. Subjects then started the next trial themselves to give them time to ask questions if they had some. The training phase lasted about $5 \mathrm{~min}$.

\section{STIMULI}

The stimuli used as the cue consisted of six letters ( L, R, D, V, W, X; see above). The letters were presented in pairs of two in the center of the screen. They were presented in white on a black background.
The auditory pacing signals were sine tones $(600 \mathrm{~Hz} ; 100 \mathrm{~ms}$ in duration) presented at a constant interval of $1000 \mathrm{~ms}$ after the CTI at a loudness level of $80 \mathrm{dBA}$. Stimulus presentation, synchronization of stimulus presentation, image pulse acquisition and recordings of motor responses was carried out with the software package Presentation (http://www.neurobs.com).

\section{MRI SCANNING PROCEDURE}

The experiment was carried out on a 3T scanner (Siemens, Erlangen, Germany). Twenty-four axial slices were acquired $(19.2 \mathrm{~cm}$ field of view, $64 \times 64$ matrix, $4 \mathrm{~mm}$ thickness, $25 \%$ gap) parallel to the AC-PC-axis and covering the whole-brain. Slice gaps were interpolated to generate output data with a spatial resolution of $3 \mathrm{~mm} \times 3 \mathrm{~mm} \times 3 \mathrm{~mm}$. A single shot, gradient recalled echo planar imaging (EPI) sequence was used (repetition time $2000 \mathrm{~ms}$, echo time $30 \mathrm{~ms}, 90^{\circ}$ flip-angle, acquisition bandwidth $100 \mathrm{kHz}$ ). Prior to functional runs corresponding 24 anatomical MDEFT slices and $24 \mathrm{EPI}_{1} \mathrm{~T}_{1}$ slices were acquired with the same geometrical parameters (slices, resolution) and the same bandwidth as used for the fMRI data. Stimuli were displayed by an LCD projector on a backprojection screen mounted in the bore of the magnet behind the participants' head.

\section{fMRI DATA ANALYSIS}

Data processing was performed using the software package LIPSIA (Lohmann et al., 2001). This software package contains tools for pre-processing, co-registration, statistical evaluation, and visualization of fMRI data. First, functional data were motion-corrected offline with the Siemens motion correction protocol (Siemens, Erlangen, Germany). Then, a sinc-interpolation algorithm was applied to correct for the temporal offset between the slices acquired in one scan. Data were filtered with a spatial Gaussian filter with $5.65 \mathrm{~mm}(\sigma=0.8)$ full width at half maximum (FWHM). A temporal high-pass filter of $90 \mathrm{~s}$ was used for baseline correction of the signal. All functional data sets were individually registered into 3-D space using participant's individual high-resolution anatomical images. This 3-D reference data set was acquired for each 
participant during a previous scanning session. The 2-D anatomical MDFET slices, geometrically aligned with the functional slices, were used to compute a transformation matrix containing rotational and translational parameter, which register the anatomical slices with the 3-D reference T1 data set. These transformation matrices were normalized to the standard Talairach stereotactic space (Talairach and Tournoux, 1988) by linear scaling and finally applied to the individual functional data. The statistical evaluation was based on a least-squares estimation using the general linear model for serially autocorrelated observations (Friston et al., 1995; Worsley and Friston, 1995). The design matrix for event-related analysis was created using a model of the hemodynamic response with a variable delay. Brain activations were analyzed in an event-related design. The four experimental conditions were modeled for cue and target as separate regressors with the trigger set on the appearance of the cue and the button press respectively (duration of analyzed events: $1000 \mathrm{~ms}$ ). Furthermore CTI and ITI were also modeled as separate regressors with the trigger set on the start of the CTI and ITI. Here the duration of the events was identical with the respective CTI and ITI duration. Only correct trials, that is, trials with correct button press $(\mathrm{L} / \mathrm{R})$ and action times [time of key press (ms) before or after onset of the tone] within the time window between $250 \mathrm{~ms}$ before and $250 \mathrm{~ms}$ after the tone were retained for further analysis. The model equation, including the observation data, the design matrix and the error term, was convolved with a Gaussian kernel with a dispersion of 4-s FWHM to account for the temporal autocorrelation (Worsley and Friston, 1995). Contrast maps were generated for each participant. As the individual functional datasets were all aligned to the same stereotactic reference space, a group analysis was then performed. A one-sample $T$-test of contrast maps across participants (random effects model) was computed to indicate whether observed differences between conditions were significantly different from 0 . Subsequently, $T$-values were transformed into $z$-scores. Results were corrected for multiple comparisons using a combination of individual voxel probability thresholding and minimum cluster size thresholding (Forman et al., 1995; Xiong et al., 1995). Given an original significance threshold of $z=3.09$ (uncorrected), 1000 iterations of Monte Carlo simulations were used to confirm that the true false-positive probability of $p=0.001$ corresponds to a minimum cluster size of $1134.00 \mathrm{~mm}^{3}$.

For the signal strength analysis we defined a mask around each region with a radius of $9 \mathrm{~mm}$. Within each subject and region (Voxel) a mean contrast was calculated separately for each condition. The mean values of the regions subsequently entered SPSS for further analysis as described in the results part.

\section{ANALYSIS OF BEHAVIORAL DATA}

As for the fMRI analysis, only correct trials were retained for further analysis. To test for differences between conditions we ran a repeated measures ANOVA with factor condition. Moreover, for each condition we ran a one-sampled $T$-test against the point of perfect synchrony $(=0 \mathrm{~ms})$.

\section{RESULTS \\ BEHAVIORAL DATA}

Subjects performed the task with high accuracy. The timing (correct tone as well as valid action time) was correct in $96.92 \%$
$(\mathrm{SD}=3.35 \%)$ of all trials. Button presses were correct in $99.15 \%$ of all trials $(\mathrm{SD}=1.104 \%)$. In the 'selection internal' conditions subjects pressed the left button more often than the right button (53.4\% compared to $46.6 \%$; paired-samples $T$-test: $t(13)=-3.066$; $p=0.009)$. In the 'timing internal' conditions subjects pressed the buttons more often with the third tone than with the fourth tone (56.77\% compared to 43.23\%; paired-samples $T$-test: $t(13)=2.646$; $p=0.020)$. Our subjects were thus slightly biased. This is in correspondence with the common finding that people cannot generate sequences that are really random.

Subjects showed in all conditions a negative asynchrony, i.e., they performed the key press slightly in advance of the target tone ['both internal': $-38.84 \mathrm{~ms}$ (SE 14.97); $t(13)=-2.594 ; p=0.022$; 'timing internal': $-39.66 \mathrm{~ms}(\mathrm{SE} 15.76) ; t(13)=-2.517 ; p=0.026$; 'selection internal': $-41.37 \mathrm{~ms}(\mathrm{SE} 15.11) ; t(13)=-2.737 ; p=0.017$; 'both external': $-45.66 \mathrm{~ms}$ (SE 14.61); $t(13)=-3.126 ; p=0.008$ ], a common finding in synchronization experiments (Aschersleben and Prinz, 1995). This shows that subjects did not simply react to the tones. However, there were no significant differences in action times between the four conditions $[F(3,11)=1.077 ; p=0.399]$.

\section{fMRI DATA \\ Whole-brain analysis}

To analyze activity contingent on the cue and activity contingent on the target we computed two separate ANOVAs with the factors action selection (internal, external) and action timing (internal, external).

Cue-related activity. We found stronger activations for internal action selection than for external action selection in the right RCZ extending to medial BA 8 (Figure 2A) and in the right precuneus (BA 7) (Table 1). We did not find any activation that was significantly stronger for external action selection than for internal action selection.

Stronger activations for internal action timing than for external action timing were found in the left superior frontal gyrus (SFG) in BA 6 that extended from the medial wall to the lateral convexity of the SFG, hereinafter referred to as paramedian frontal cortex (Figure 3A; Table 1). Additionally we found activations that were stronger for external action timing than for internal action timing. These were located bilaterally at the border between inferior occipital lobe and fusiform gyrus (Table 1). Finally, the interaction of action timing and action selection did not yield any activation that survived the cluster threshold.

Target-related activity. Although our main focus was on activations appearing around the point in time when subjects made their decisions (i.e., on cue-related activation) we also analyzed the processes taking place around the implementation of the decision. Stronger activations for internal action selection than for external action selection were found bilaterally in the middle frontal gyrus (BA 10), in the inferior parietal lobe (BA 40), as well as at the border between inferior frontal gyrus (BA 47) and the insula (BA 13) (see Table 2). The reverse comparison revealed no activation that survived the threshold. When computing the main effect for the when component, we found only an activation that was stronger for external action timing than for internal action timing. This was located in the lingual gyrus (BA 18). 


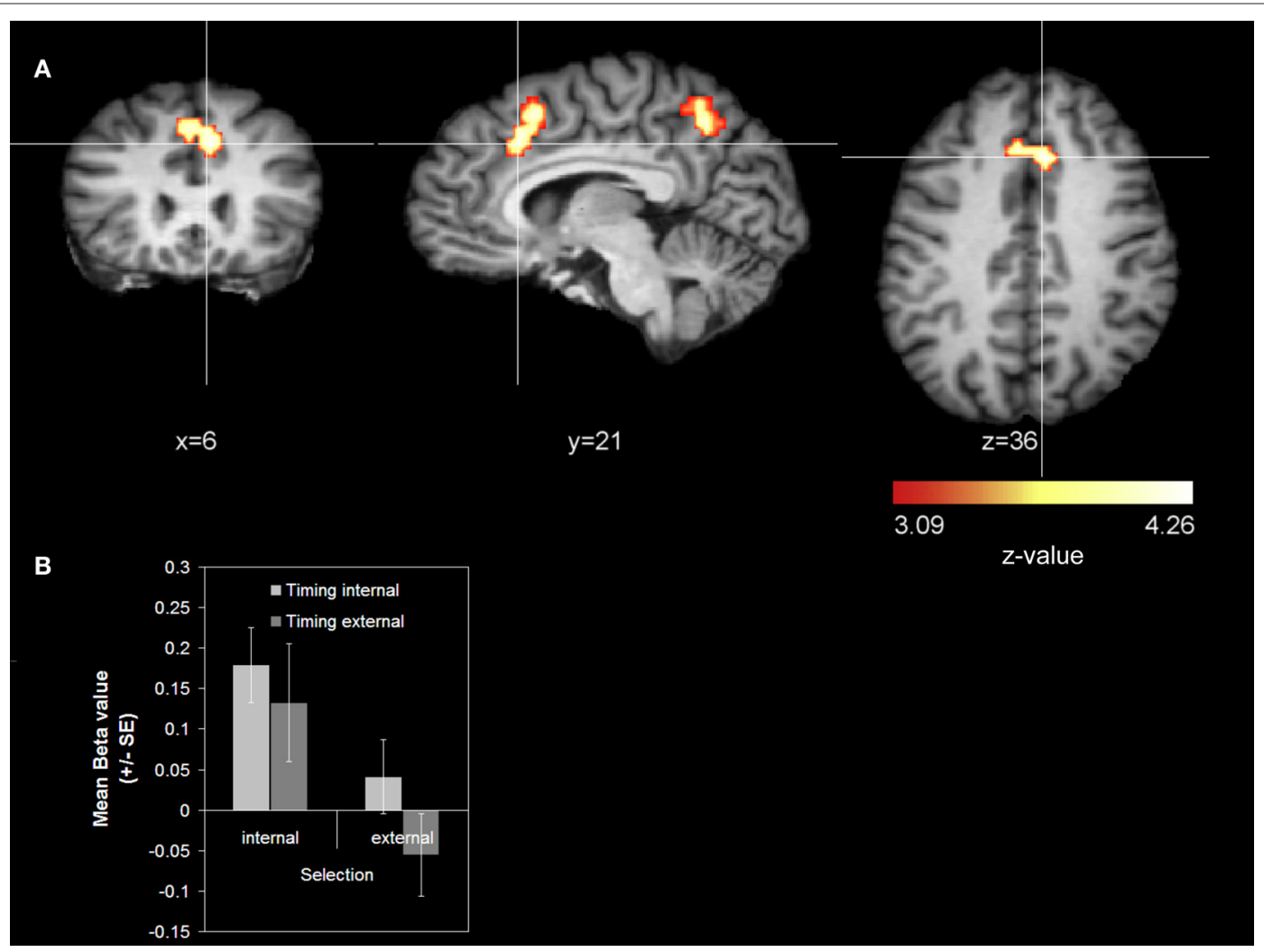

FIGURE 2 | Main effect action selection (cue-related activity). (A) Whole-brain analysis. The contrast was averaged over 14 subjects ( $z$-threshold at $z=3.09$, corrected) and mapped to an individual brain from the in-house database. The comparison internal action selection > external action selection is shown. (B) Signal strength analysis. The diagram reports mean beta values for the RCZ coordinate $(x=6, y=21, z=36)$.

Table $\mathbf{1}$ |Anatomical location, hemisphere, maximum $Z$ value $(\boldsymbol{p}=\mathbf{0 . 0 0 1}$, corrected) and Talairach coordinates. Cue-related activations with a minimum cluster size of $1134 \mathrm{~mm}^{3}$ are shown.

\begin{tabular}{|c|c|c|c|c|c|}
\hline \multirow[t]{2}{*}{ Anatomical area } & \multirow[t]{2}{*}{ Side } & \multirow[t]{2}{*}{$Z_{\max }$} & \multicolumn{3}{|c|}{ Talairach coordinates } \\
\hline & & & $x$ & $y$ & $z$ \\
\hline \multicolumn{6}{|l|}{ Internal > external } \\
\hline Rostral cingulate zone & $\mathrm{R}$ & 4.26 & 6 & 21 & 36 \\
\hline \multicolumn{6}{|l|}{ Internal > external } \\
\hline Superior frontal Gyrus (paramedian frontal cortex) & $\mathrm{L}$ & 4.48 & -18 & 12 & 51 \\
\hline \multicolumn{6}{|l|}{ External > internal } \\
\hline Inferior occipital lobe/fusiform gyrus & $\mathrm{L}$ & 4.95 & -42 & -66 & -3 \\
\hline
\end{tabular}

We observed no activations in the frontomedian wall, neither for the main effect action selection, nor for the main effect action timing. However, if we lowered the threshold to a more liberal one $(z>3.09$; $\mathrm{p}=0.05$, corrected) we observed an activation in the RCZ for the main effect action selection. However, this activation was much smaller compared to the cue-related activation $\left(567 \mathrm{~mm}^{3}\right.$ compared to $1944 \mathrm{~mm}^{3}$ ). As for the cue-related activation we did not find any activation that survived the cluster threshold when computing the interaction term of action selection and action timing.

Signal strength analysis. To gain more detailed information about the pattern of activation of the cue-related activations that were linked to the two voluntary action components in the whole-brain analysis we performed a signal strength analysis in the two main 


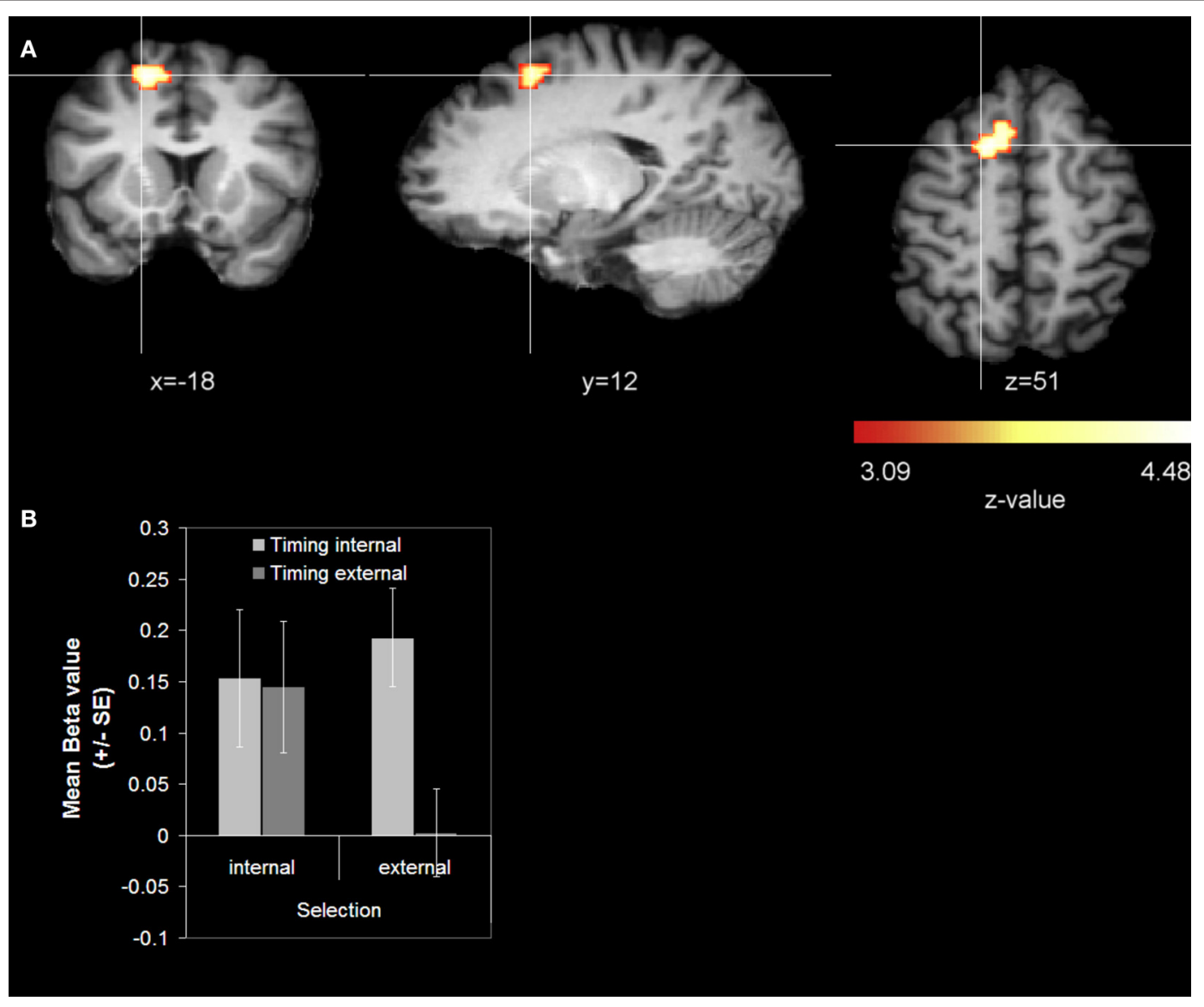

FIGURE 3 | Main effect action timing (cue-related activity). (A) Whole-brain analysis. The contrast was averaged over 14 subjects ( $z$-threshold at $z=3.09$, corrected) and mapped to an individual brain from the in-house database. The comparison internal action timing > external action timing is shown. (B) Signal strength analysis. The diagram reports mean beta values for the SFG coordinate $(x=-18, y=12, z=51)$.

Table 2 |Anatomical location, hemisphere, maximum $Z$ value $(\boldsymbol{p}=\mathbf{0 . 0 0 1}$, corrected) and Talairach coordinates. Target-related activations with a minimum cluster size of $1134 \mathrm{~mm}^{3}$ are shown.

\begin{tabular}{|c|c|c|c|c|c|}
\hline \multirow[t]{2}{*}{ Anatomical area } & \multirow[t]{2}{*}{ Side } & \multirow[t]{2}{*}{$Z_{\max }$} & \multicolumn{3}{|c|}{ Talairach coordinates } \\
\hline & & & $x$ & $y$ & $z$ \\
\hline \multicolumn{6}{|l|}{ Internal > external } \\
\hline Middle frontal gyrus (BA 10) & $\mathrm{R}$ & 4.17 & 30 & 51 & 21 \\
\hline Inferior parietal lobe (BA 40) & $\mathrm{R}$ & 3.89 & 48 & -36 & 51 \\
\hline Inferior parietal lobe (BA 40) & $\mathrm{L}$ & 5.28 & -51 & -42 & 48 \\
\hline \multicolumn{6}{|l|}{ MAIN EFFECT ACTIONTIMING } \\
\hline \multicolumn{6}{|l|}{ External > internal } \\
\hline Lingual gyrus (BA 18) & $\mathrm{L}$ & -4.90 & -9 & -72 & -3 \\
\hline
\end{tabular}

clusters for internal action selection (RCZ) and internal action timing (paramedian frontal cortex). A repeated measures ANOVA with factors action selection (internal vs. external) and action timing (internal vs. external) was computed for each region.
For the RCZ the results replicated the findings of the wholebrain analysis showing a significant main effect for action selection (internal > external) $[F(1,13)=15.456 ; p=0.002]$ (see Figure 2B). The main effect action timing (internal $>$ external) was not 
significant $[F(1,13)=3.77 ; p=0.074]$. For the paramedial frontal cortex the results showed a significant main effect action timing (internal > external) $[F(1,13)=16.808 ; p=0.001]$, while the main effect action selection was not significant $[F(1,13)=3.016$; $p=0.104]$. Additionally there was a significant interaction of action selection $\times$ action timing $[F(1,13)=8.151 ; p=0.014]$. Only when the action selection was externally, internal action timing resulted in a stronger activation than external action timing (see Figure 3B).

However, it is important to note that the results of the signal strength analysis were obtained post hoc and are based on a much more liberal threshold than the whole-brain analysis and therefore should be interpreted with care.

\section{DISCUSSION}

The aim of the present study was to dissociate the 'when-' and the 'what-component' of intentional action on the functional neuroanatomical level. In order to do so, participants were instructed to perform one of two possible actions at one of two possible points in time. We varied whether participants freely chose between the two actions and the two points in time, or whether action and point in time were indicated by a stimulus. In other words, 'what' and/or 'when' of the to-be-produced action (selection and timing) were based either on some internal decision or were specified by an external stimulus.

We were primarily interested in activation differences underlying the decisional aspects of voluntary action that is reflected in the BOLD response contingent on the cue. The fact that we observed different activation maxima for internal action selection and internal action timing in decision relevant areas of the frontomedian wall stresses the fact that subjects really decided on the cue. The whole-brain analysis revealed two areas in the frontomedian wall that fulfill different functions. The RCZ is involved in the internal selection of an action, whereas an area of the SFG in the left paramedian frontal cortex is involved in the decision ' $w$ hen' to perform an action. However, the signal strength analysis revealed an interaction of the action timing and action selection in the paramedian frontal cortex. When action selection was internally specified the activation level was equally high for internal and external action timing. Only when action selection was externally specified the activation level in the paramedian frontal cortex differed between internal and external action timing.

Notice that we found the strongest differential activations in the RCZ and paramedian frontal cortex in the analysis of the cuerelated signal and not in the analysis of the target-related signal, indicating that these activations reflect processes preparing the upcoming action (viz. the internal specification of the when and what action parameters) and not processes related to the execution of the action.

\section{THE ROLE OF THE RCZ IN INTERNALLY SELECTED ACTIONS}

The finding that the RCZ is primarily involved in internal action selection, but not in internal action timing confirms the results from Mueller et al. (2007). These authors demonstrated increased RCZ activity for internally selected actions as compared to externally selected actions, while keeping the timing of the action constant. While involvement of the RCZ in internal action selection has also been found in several other studies (Cunnington et al., 2003, 2006; Deiber et al., 1999; Lau et al., 2004b; van Eimeren et al., 2006; Wiese et al., 2004, 2005) the present study is to our knowledge the first that shows that this region is more sensitive for a selection manipulation than for a timing manipulation.

The functional role of the RCZ in voluntary action selection is still under discussion. Walton et al. (2004) argue that RCZ activity is related to the internal selection of an action and to assessing the consequences of the chosen action. This notion fits to the ideomotor principle of action control (Herwig et al., 2007; Hommel et al., 2001; James, 1890, 1950; Prinz, 1997; Waszak and Herwig, 2007) that assumes that action representations are activated by anticipation of the actions' effect, including body-related afferent information, that is, proprioceptive feedback (Hommel et al., 2001). The view of representing actions in terms of their action goals is widely spread (Jeannerod, 1999; Rizzolatti et al., 1997).

On the other hand it has been argued that the RCZ might be involved in conflict monitoring (Botvinick et al., 2001, 2004; Carter et al., 1998; Lau et al., 2004b, 2006) rather then in voluntary selection of action. Lau et al. $(2006,2004 b)$ argue that, if a response has to be selected randomly from a set of possible responses, the response is underdetermined and, therefore, triggers response conflict.

We believe that both interpretations are complimentary rather than contradictory. Selecting one response implies to reject all alternative responses. This idea is not new, but already expressed by James $(1890,1950)$ who said 'The express fiat, or act of mental consent to the movement, comes in when the neutralization of the antagonistic and inhibitory idea is required' (p. 526). The less the selection is predetermined, the more the alternative responses interfere with the selection and the more internal effort is required (Forstmann et al., 2006). This idea is in accordance with the suggestion from Brass and Haggard (2008) that response conflict is an inherent property of all action.

In addition to the hypothesized activation in the RCZ we found increased activation in the posterior precuneus that was related to internal action selection. The precuneus is extensively connected with the RCZ (Leichnetz, 2001; Petrides and Pandya, 1984). Furthermore there is evidence that the precuneus plays a role in voluntary action. In a recent neuroimaging study, Soon et al. (2008) found the anterior precuneus - in addition to the frontopolar cortex - to be a predictor of subjects' free decisions ahead of time.

\section{THE ROLE OF THE SUPERIOR FRONTAL GYRUS IN INTERNALLY TIMED ACTIONS}

Activation in a part of the left SFG in the paramedian frontal cortex was found to be stronger for internally timed than for externally timed actions. This region is located slightly anterior and lateral to the preSMA. This is to our knowledge the first evidence for an involvement of the SFG in the 'when-component' of intentional action. At first sight this finding seems to be at odds with studies that indicated the preSMA to be involved in the decision when to act (Cunnington et al., 2002, 2003; Deiber et al., 1999; Jahanshahi et al., 1995; Jenkins et al., 2000; Wiese et al., 2004, 2005). However, in contrast to the present study, these studies did not disentangle processes related to the decision when to act from processes related to the instantaneous initiation of the action and therefore 
presumably confounded these two factors. Moreover, the activation we found is located very close to the preSMA. The existence of a functional link between paramedian frontal cortex and preSMA is thus probable.

The results from the signal strength analysis extend the results from the whole-brain analysis by showing an additional interaction between selection and timing manipulation. Thus it seems that the paramedial frontal cortex is stronger activated for internal than for external action timing only if action selection is externally specified. If action selection is internally specified, paramedian frontal activation is almost the same for internal and external action timing.

Other structures that are considered to be involved in the timing of actions are the basal ganglia and the cerebellum (e.g., Elsinger et al., 2006; Jahanshahi et al., 2006; Taniwaki et al., 2003). We did not observe activation differences in these structures contingent on the cue, probably because they are not involved in the decision process (that takes place right after cue presentation), but only in the implementation of the action (that takes place during the target period). Furthermore we did not observe activation differences in the basal ganglia and cerebellum during the target period either, probably because at this processing stage both internally and externally timed actions draw equally on these structures.

\section{DIFFERENTIATING WHAT- AND WHEN-COMPONENT OF INTENTIONAL ACTIONS}

Our findings suggest the existence of at least partially dissociable neural networks underlying the 'what-' and 'when-component' of intentional action: the RCZ and paramedian frontal cortex are differentially activated for intentional action selection and action timing, respectively. Nonetheless, the interaction between action timing and action selection within the paramedian frontal cortex revealed by the signal strength analysis indicates that decisional processes concerning action timing and selection are not completely dissociated. This finding is not surprising in the light of the functional interdependency of these two components of voluntary action control: For an action and its consequences to be evaluated both components have to be taken into account. This interdependency can be illustrated by looking at the example of the soccer player who has to decide whether to pass the ball or to shoot on the goal. His choice strongly depends on the moment when he intends to act. The optimal timing, in turn, depends largely on the action he chooses.

The nature of the interdependency between internal action selection and timing remains subject for future research. Deecke (1996) suggested a hierarchical organization with 'what' and 'how' decision preceding the 'when-decision'. Although we have not investigated the sequential order in which the two decisions are taken, we consider our data to be in line with a superordinate role of the 'what'-decision that is subsequently timed and integrated into an action plan.

\section{ACTIVATIONS RELATED TO THE TARGET}

Although the present study focused mainly on decisional processes of voluntary action control taking place contingent on the presentation of the cue, activations related to the target tone should be considered briefly.
The activations found for the main effect action selection are similar to those found in prospective memory tasks such as the middle frontal gyrus (BA 10) and the inferior parietal lobe (BA 40) (c.f. Simons et al., 2006). In event-based prospective memory the intended action has to be remembered until the intention is retrieved upon presentation of the triggering stimulus condition (c.f. Einstein et al., 1992). Evidently, in our task subjects have to remember the selected action parameters until execution. Our results seem to indicate that the retrieval of internally generated action intentions draws especially heavily on the prospective memory network. This may be due to the quality of a representation specified by some distinct external stimulus being better than the quality of an internally generated representation. That this effect was observed for internally selected actions only and not for internally timed actions may be due the fact that, concerning the timing component of our task, the to-be-retrieved action parameter (which tone) and 'retrieval cue' (target tone) are basically identical.

Most important in the present context is the absence of targetrelated activation differences within the frontomedian wall. Only when the threshold was lowered we could observe activation in the RCZ for the main effect action selection. However, compared to the cue-related activation this activation has a much smaller extend. Given the above mentioned stronger involvement of prospective memory in the implementation of internal action selection the activation in the RCZ might reflect processes related to the updating of the decision. This finding confirms the notion that activation differences in the frontomedian wall reflect primarily decisional aspects of voluntary action control, rather than processes related to the implementation of the action.

\section{CONCLUSIONS}

The aim of the present study was to experimentally differentiate between different components of intentional action. Our experiment shows that the intentional decision when to execute an action is associated with activation in a part of the SFG, the paramedian frontal cortex, while the intentional decision which action to execute activates the RCZ.

Thus, we were able to dissociate the processes underlying 'what' and 'when' based voluntary decision processes. This challenges the idea of a unitary system supporting voluntary action. Instead, we propose that voluntary action control is an interplay of different neuroanatomically dissociable subfunctions. It is conceivable that in addition to the when- and what-component of intentional action other components might be dissociable as well (Brass and Haggard, 2007, 2008). Such a functional segregation of intentional action will certainly help to understand the confusing pattern of empirical findings. Furthermore, it is consistent with philosophical conceptions of intentions related to action which also distinguish different levels of action-related intentions.

\section{ACKNOWLEDGEMENTS}

We thank Joeran Lepsien for support with the fMRI-design. Florian Waszak was supported by a grant from the Agence National de la Recherche (ANR-08-FASHS-013). 


\section{REFERENCES}

Aschersleben, G., and Prinz, W. (1995). Synchronizing actions with events: the role of sensory information. Percept. Psychophys. 57, 305-317.

Blakemore, S. J., Wolpert, D. M., and Frith, C. D. (1998). Central cancellation of self-produced tickle sensation. Nat. Neurosci. 1, 635-640.

Botvinick,M.M.,Braver, T.S.,Barch, D. M., Carter, C. S., and Cohen, J. D. (2001). Conflict monitoring and cognitive control. Psychol. Rev. 108, 624-652.

Botvinick, M. M., Cohen, J. D., and Carter, C.S. (2004). Conflict monitoring and anterior cingulate cortex: an update. Trends Cogn. Sci. 8, 539-546.

Brass, M., and Haggard, P. (2007). To do or not to do: the neural signature of selfcontrol. J. Neurosci. 27, 9141-9145.

Brass, M., and Haggard, P. (2008). The what, when, whether model of intentional action. Neuroscientist 14, 319-325.

Carter, C. S., Braver, T. S., Barch, D. M., Botvinick, M. M., Noll, D., and Cohen, J. D. (1998). Anterior cingulate cortex, error detection, and the online monitoring of performance. Science 280, 747-749.

Cunnington, R., Windischberger, C., Deecke, L., and Moser, E. (2002). The preparation and execution of selfinitiated and externally-triggered movement: a study of event-related fMRI. Neuroimage 15, 373-385.

Cunnington, R., Windischberger, C., Deecke, L., and Moser, E. (2003). The preparation and readiness for voluntary movement: a highfield event-related fMRI study of the Bereitschafts-BOLD response. Neuroimage 20, 404-412.

Cunnington, R., Windischberger, C., and Moser, E. (2005). Premovement activity of the pre-supplementary motor area and the readiness for action: studies of time-resolved event-related functional MRI. Hum. Mov. Sci. 24, 644-656.

Cunnington, R., Windischberger, C., Robinson, S., and Moser, E. (2006). The selection of intended actions and the observation of others' actions: a time-resolved fMRI study. Neuroimage 29, 1294-1302.

Debaere, F., Wenderoth, N., Sunaert, S., Van Hecke, P., and Swinnen, S. P. (2003). Internal vs external generation of movements: differential neural pathways involved in bimanual coordination performed in the presence or absence of augmented visual feedback. Neuroimage 19, 764-776.

Deecke, L. (1996). Planning, preparation, execution, and imagery of volitional action. Brain Res. Cogn. Brain Res. 3, 59-64.
Deiber, M. P., Honda, M., Ibanez, V., Sadato, N., and Hallett, M. (1999). Mesial motor areas in self-initiated versus externally triggered movements examined with fMRI: effect of movement type and rate. J. Neurophysiol. 81, 3065-3077.

Einstein, G. O., Holland, L. J., McDaniel, M. A., and Guynn, M. J. (1992). Age-related deficits in prospective memory: the influence of task complexity. Psychol. Aging 7, 471-478.

Elsinger, C. L., Harrington, D. L., and Rao, S. M. (2006). From preparation to online control: reappraisal of neural circuitry mediating internally generated and externally guided actions. Neuroimage 31, 1177-1187.

Forman, S. D., Cohen, J. D., Fitzgerald, M., Eddy, W. F., Mintun, M. A., and Noll, D. C. (1995). Improved assessment of significant activation in functional magnetic resonance imaging (fMRI): use of a cluster-size threshold. Magn. Reson. Med. 33, 636-647.

Forstmann, B. U., Brass, M., Koch, I., and von Cramon, D. Y. (2006). Voluntary selection of task sets revealed by functional magnetic resonance imaging. J. Cogn. Neurosci. 18, 388-398.

Friston, K. J., Holmes, A. P., Poline, J. B., Grasby, P. J., Williams, S. C., Frackowiak, R. S., and Turner, R. (1995). Analysis of fMRI time-series revisited. Neuroimage 2, 45-53.

Goldberg, G. (1985). Supplementary motor area structure and function: review and hypotheses. Behav. Brain Sci. 8, 567-616.

Greenwald, A. (1970). Sensory feedback mechanisms in performance control: with special reference to the ideomotor mechanism. Psychol. Rev. 77, 73-99.

Haggard, P., and Clarke, S. (2003). Intentional action: conscious experience and neural prediction. Conscious. Cogn. 12, 695-707.

Herwig, A., Prinz, W., and Waszak, F. (2007). Two modes of sensorimotor integration in intention-based and stimulus-based actions. Q. J. Exp. Psychol. 60, 1540-1554.

Hommel, B. (2000). The prepared reflex: automaticity and control in stimulus-response translation. In Control of Cognitive Processes: Attention and Performance, Vol. XVIII, S. Monsell and J. Driver, eds (Cambridge, MA, MIT Press), pp. 247-273.

Hommel, B., Müsseler, J., Aschersleben, G., and Prinz, W. (2001). The theory of event coding (TEC): a framework for perception and action planning. Behav. Brain Sci. 24, 849-937.
Jahanshahi, M., and Frith, C. D. (1998). Willed action and its impairments. Cogn. Neuropsychol. 15, 483-533.

Jahanshahi,M., Jenkins, I.H., Brown, R. G., Marsden, C. D., Passingham, R. E., and Brooks, D. J. (1995). Self-initiated versus externally triggered movements. I. An investigation using measurement of regional cerebral blood flow with PET and movement-related potentials in normal and Parkinson's disease subjects. Brain 118, 913-933.

Jahanshahi,M.,Jones, C.R., Dirnberger, G., and Frith, C. D. (2006). The substantia nigra pars compacta and temporal processing. J. Neurosci. 26 12266-12273.

James, W. (1890, 1950). The Principles of Psychology. New York, Dover Publications.

Jeannerod, M. (1999). The 25th Bartlett lecture. To act or not to act: perspectives on the representation of actions. Q. J. Exp. Psychol. A 52, 1-29.

Jenkins, I.H., Jahanshahi, M., Jueptner, M., Passingham, R. E., and Brooks, D. J. (2000). Self-initiated versus externally triggered movements. II. The effect of movement predictability on regional cerebral blood flow. Brain $123,1216-1228$

Lau, H., Rogers, R. D., and Passingham, R. E.(2006). Dissociating response selection and conflict in the medial frontal surface. Neuroimage 29, 446-451.

Lau, H. C., Rogers, R. D., Haggard, P., and Passingham, R. E. (2004a). Attention to intention. Science 303, 1208-1210.

Lau, H. C., Rogers, R. D., Ramnani, N., and Passingham, R. E. (2004b). Willed action and attention to the selection of action. Neuroimage 21, 1407-1415.

Leichnetz, G. R. (2001). Connections of the medial posterior parietal cortex (area 7m) in the monkey. Anat. Rec. 263, 215-236.

Lohmann, G., Müller, K., Bosch, V., Mentzel, H., Hessler, S., Chen, L., Zysset, S., and von Cramon, D. Y. (2001). LIPSIA - a new software system for the evaluation of functional magnetic resonance images of the human brain. Comput. Med. Imaging Graph. 25, 449-457.

Mueller, V. A., Brass, M., Waszak, F., and Prinz, W. (2007). The role of the preSMA and the rostral cingulate zone in internally selected actions. Neuroimage 37, 1354-1361.

Oldfield, R. C. (1971). The assessment and analysis of handedness: the Edinburgh inventory. Neuropsychologia 9, 97-113.

Petrides, M., and Pandya, D. N. (1984). Projections to the frontal cortex from the posterior parietal region in the rhesus monkey. J. Comp. Neurol. 228 105-116.

Prinz, W. (1997). Perception and action planning. Eur. J. Cogn. Psychol. 9, 129-154.

Rizzolatti, G., Fogassi, L., and Gallese, V. (1997). Parietal cortex: From sight to action. Curr. Opin. Neurobiol. 7, 562-567.

Rushworth, M. F., Walton, M. E., Kennerley, S.W., andBannerman, D. M (2004). Action sets and decisions in the medial frontal cortex. Trends Cogn. Sci. 8, 410-417.

Simons,J.S.,Schölvinck, M.L., Gilbert, S. J. Frith, C. D., and Burgess, P.W. (2006). Differential components of prospective memory? Evidence from fMRI. Neuropsychologia 44, 1388-1397.

Soon, C. S., Brass, M., Heinze, H. J., and Haynes, J. D. (2008). Unconscious determinants of free decisions in the human brain. Nat. Neurosci. 11, 543-545.

Talairach, J., and Tournoux, P. (1988). Co-Planar Stereotaxic Atlas of the Human Brain, 3-Dimensional Proportional System: An Approach to Cerebral Imaging. New York/ Stuttgart, Thieme Medical Publisher/ Georg Thieme Verlag.

Taniwaki, T., Okayama, A., Yoshiura, T., Nakamura, Y., Goto, Y., Kira, J., and Tobimatsu, S. (2003). Reappraisal of the motor role of basal ganglia: a functional magnetic resonance image study. J. Neurosci. 23, 3432-3438.

van Eimeren, T., Wolbers, T., Munchau, A., Buchel, C., Weiller, C., and Siebner, H. R. (2006). Implementation of visuospatial cues in response selection. Neuroimage 29, 286-294.

Walton, M. E., Devlin, J. T., and Rushworth, M. F. (2004). Interactions between decision making and performance monitoring within prefrontal cortex. Nat. Neurosci. 7, 1259-1265.

Waszak, F., and Herwig, A. (2007). Effect anticipation modulates deviance processing in the brain. Brain Res. 1183, 74-82.

Waszak, F., Wascher, E., Keller, P., Koch, I., Aschersleben, G., Rosenbaum, D. A. and Prinz, W. (2005). Intention-based and stimulus-based mechanisms in action selection. Exp. Brain Res. 162, 346-356.

Wiese, H., Stude, P., Nebel, K., de Greiff, A., Forsting, M., Diener, H. C., and Keidel, M. (2004). Movement preparation in self-initiated versus externally triggered movements: an event-related fMRI-study. Neurosci. Lett. 371, 220-225.

Wiese, H., Stude, P., Nebel, K., Forsting, M., and de Greiff, A. (2005). Prefrontal cortex activity in self-initiated 
movements is condition-specific, but not movement-related. Neuroimage 28, 691-697.

Worsley, K. J., and Friston, K. J. (1995). Analysis of fMRI time-series revisited - again. Neuroimage 2, 173-181.

Xiong, J., Goa, J.-H., Lancaster, J. L., and Fox, P. T. (1995). Clustered pixels analysis for functional MRI activation studies of the human brain. Hum. Brain Mapp. 3, 287-301.

Conflict of Interest Statement: The authors declare that the research was conducted in the absence of any commercial or financial relationships that could be construed as a potential conflict of interest.
Received: 24 November 2008; paper pending published: 01 January 2009; accepted: 14 February 2009; published online: 26 February 2009.

Citation: Krieghoff V, Brass M, Prinz W and Waszak F (2009) Dissociating what and when of intentional actions. Front. Hum. Neurosci. (2009) 3:3. doi: 10.3389/neuro.09.003.2009
Copyright () 2009 Krieghoff, Brass, Prinz and Waszak. This is an open-access article subject to an exclusive license agreement between the authors and the Frontiers Research Foundation, which permits unrestricted use, distribution and reproduction in any medium, provided the original authors and source are credited. 